\title{
IWOR: tribute to Professor Laureano F. Escudero on occasion of his 65th birthday
}

\author{
Antonio Alonso-Ayuso • Joaquín Sánchez-Soriano
}

Published online: 7 May 2009

(C) Sociedad de Estadística e Investigación Operativa 2009

The International Workshop on Operational Research (IWOR) conference held in Madrid (5-7 June 2008) was intended to be a tribute to the outstanding professional and scientific career in Operational Research of Professor Laureano F. Escudero on the occasion of his 65th birthday. During the conference, the participants presented more than 100 papers on many different topics in Operational Research. Furthermore, six plenary lectures were delivered by Brenda Dietrich (Math at IBM), Rüdiger Schultz (Dominance Constraints in Two-stage Stochastic Programming), Gautam Mitra (Downside Risk and Portfolio Models), Monique Guignard-Spielberg (Primal and Convex Hull Relaxations for Nonlinear Integer Programming Problems), Maarten H. van der Vlerk (Some Approaches to Solving Stochastic Mixed-Integer Programs), and Martin Gröstschel (Controlling Paths), which are related to several of the topics in which Professor Escudero contributed.

This special issue of TOP has a double purpose. On the one hand, it contains a collection of selected papers from an open call for papers on Operational Research topics, and, on the other hand, it constitutes an additional tribute to Professor Laureano F. Escudero.

Since this special issue is devoted to the figure of Professor Laureano F. Escudero, before going to summarise the contents of it, we provide a brief biography of him.

Laureano F. Escudero Bueno (September 23rd, 1942) is PhD in Economic Sciences and currently full professor at the Department of Statistics and Operational Research at the Rey Juan Carlos University (Madrid, Spain, since 2007). Previously, he

\footnotetext{
A. Alonso-Ayuso ( $\square)$

Universidad Rey Juan Carlos, 28033 Mostoles, Madrid, Spain

e-mail: antonio.alonso@urjc.es

J. Sánchez-Soriano

Universidad Miguel Hernández de Elche, 03202 Elche, Alicante, Spain
} 
has been professor of Statistics and Operational Research at the Complutense University of Madrid (Spain, 1992-2000) and at the Miguel Hernández University of Elche (Alicante, Spain, 2000-2007).

Other than his academic activity at the University, his scientific career has been developed in the most prestigious IBM scientific centres: Scientific Centre of Madrid (Spain), Palo Alto Scientific Centre (California, USA), German Manufacturing Technology Centre (Sindelfingen, Germany), and T.J. Watson Research Centre in Yorktown Heights (NY, USA). In the early 1990s, Laureano returned to Spain to be head of the Department of Decision Support Systems first in UITESA, and later, when both companies merged, in "Iberdrola Ingeniería y Consultoría." During the period 20032004, he was the President of EURO (The Association of the European Operational Research Societies), being the first Spaniard granted with this honour.

Professor Escudero is a leading figure in several Operational Research fields, having made seminal contributions in combinatory and integer programming, decision support models, and stochastic programming. He has been the supervisor of nine $\mathrm{PhD}$ students, has published more than 120 papers in international journals and books, and 5 books and monographs in Spanish. His figure will always be related to the development of the Operational Research in Spain during the last decades.

Prof. Escudero's current scientific work is focused on cutting plane-based tightening integer and combinatorial programs, parallel computation and stochastic programming. During the last 30 years, his application fields have been strategic and production planning modelling and logistics for the energy, water resources and manufacturing sectors.

During his fruitful professional and scholar career, he has received the following Prizes and Awards:

- In 1972 and 1976, the IBM Outstanding Contribution Award.

- In 1976, Prize to the best scientific/technical book, granted by the Spanish Engineering Institute, Vizcaya office.

- In 1986, Cross to the Aeronautical Merit, white colour, first class granted by the Spanish Government.

- In 1990, "Member of the IBM Academy of Technology."

- In 2006, The EURO Golden Pin granted by the Association of European Operational Research Societies.

Next, we present a brief summary of the contributions contained in this special issue which cover a small range of topics presented during the IWOR.

Very frequently, mainly in optimisation problems with a given time horizon to exploit, some coefficients in the objective function and the right-hand-side vector and, in a lesser extend, the constraint matrix are not known with certainty when the decisions are to be made, but some information about the parameters is available. This circumstance allows one to use Stochastic Integer Programming (SIP) for solving multistage mixed 0-1 programs under uncertainty. Escudero presents a framework for solving large-scale multistage mixed 0-1 optimisation problems under uncertainty by using SIP in his work "On a mixture of the fix-and-relax coordination and Lagrangean substitution schemes for multi-stage stochastic mixed integer programming" based on scenario analysis, branch-and-fix coordination and Lagrangean relaxations. 
In the paper "Local decision making and decision fusion in hierarchical levels" by Beldek and Leblebicioğlu, a hierarchical decision-making framework is proposed. At each level, some temporary solutions are obtained; then a suitable decision fusion technique is used to merge the temporary solutions for the next level. The proposed framework depends on reutilisation and/or elimination at each level of local agents in the previous level that together performs the decisions via a suitable decisionfusion technique. Reutilisation and elimination operations are performed according to a user-defined performance criterion: If an agent is successful in its local region, it is reutilised in the next level, otherwise it is eliminated, and new agents take its place. This framework is applied on a fault detection problem

We can find many examples in which the decisions are made by a voting procedure; in those situations knowing which coalitions are winning is relevant to analyse the power of each agent involved in the decision process. However, "blocking coalitions" may arise in such situations that, in spite of being not winning, are powerful enough to prevent a proposal to be accepted, and this capability becomes relevant in certain contexts. Carreras in his paper "Protectionism and blocking power indices" study blocking coalitions and provide different theoretical and practical results about it. In particular, he studies the protectionist role of blocking coalitions in a voting situation.

The paper "Strategic absentmindedness in finitely repeated games" by GarcíaJurado, Llorca, Meca, Pulido and Sánchez-Soriano considers finitely repeated games in which players have the possibility to behave absentminded in some parts of the game and in which they use this ability strategically. In García-Jurado et al. (2000) and García-Jurado and González-Díaz (2006), it has been shown that Folk theorems persist when players can unilaterally commit to a subset of strategies before playing a finite repeated game. In this paper it is proved that the standard conditions for folk theorems can be substantially relaxed when players can make absentmindedness compromises, both when dealing with Nash and with subgame perfect equilibrium concepts.

The general multistage mixed $0-1$ optimisation model is a mixed linear program to solve problems going through several stages. However, in many real cases the model must be extended to consider the uncertainty in some of the main parameters. Escudero, Garín, Merino and Pérez in their paper "BFC-MSMIP: an exact Branch-andFix Coordination approach for solving multistage stochastic mixed 0-1 problems" present an exact algorithmic framework for optimising multistage stochastic mixed 0-1 problems with complete recourse. An important feature of their approach with respect to other stochastic integer approaches is that it addresses multistage environments with both continuous and 0-1 variables at any stage of the time horizon. Furthermore, the computational experience shows that the new approach obtains the optimal solution in all instances under consideration.

The paper "Multi-choice clan games and their core" by Branzei, Llorca, Sanchez Soriano and Tijs, generalises clan games introduced by Potters et al. (1989) by considering that non-clan agents may exert different levels of effort when included in a coalition, while clan members can only decide whether to participate or not. This is, then, a particular class of the multi-choice cooperative games introduced by Hsiao and Raghavan (1993). The focus is on the analysis of the core. First, an explicit description of the core of a multi-choice clan game is given. Then, given a coalition $\mathrm{C}$, 
multi-choice clan games with clan $\mathrm{C}$ are characterised by the shape of the core using two sets of special allocations called the clan corner and the union corner. This characterisation allows defining a family of suitable rules on the class of multi-choice clan games that are additive and associate with each multi-choice clan game a core allocation. Further, the authors give a characterisation of the core of monotonic multichoice clan games. Finally, the connection between the convexity of the multi-choice clan game and the stability of its core is studied.

Two reinsurance treaties, so-called excess-loss and stop-loss reinsurance, that concern with portfolios have been applied in several insurance branches, where the insurer stipulates an upper limit to the amount to be paid out, and the reinsurer covers the excess over the fixed threshold. Escudero and Ortega in their paper "How retention levels influence the variability of the total risk under insurance" study how dependencies influence the variability of the retained and the reinsured total amount, under excess-loss and stop-loss reinsurance policies with stochastic retention levels.

In the paper "Applying Mod-k-Cuts for Solving Linear Ordering Problems" by Oswald, Reinelt and Seitz, the linear ordering problem (LOP) is studied. LOP is a classical NP-hard combinatorial optimisation problem with many real-world applications, including, for example, the triangulation of input-output matrices in economics, minimising total weighted completion time in one-machine scheduling or aggregation of individual preferences. The authors report in this paper the usefulness of mod-k cuts in a branch-and-cut algorithm for solving linear ordering problems to optimality.

Rodríguez, Albornoz and Pla-Aragones in their paper "A two-stage stochastic programming model for scheduling replacements in sow farms" study sow farms producing piglets in order to plan, in a medium-term horizon, the optimal replacement and schedule of purchases. They take into account different deterministic and stochastic parameters relevant for the problem under consideration. The authors describe the sow farms producing piglets problem through a suitable two-stage stochastic linear programming model with recourse. They solve this problem and compare their results to those obtained with previous deterministic models assessing the suitability of the stochastic approach.

Felipe, Ortuño and Tirado in their paper "New neighbourhood structures for the Double Travelling Salesman Problem With Multiple Stacks" propose four new neighbourhood structures for a recently introduced vehicle routing problem with precedence and loading constraints and provide a detailed mathematical description of them. Some promising computational results for real-sized instances obtained using these structures with different heuristic strategies are also presented.

Acknowledgements The guest editors are grateful to TOP and its Editors, Emilio Carrizosa and Justo Puerto, for their trust in this special issue. We are also grateful to all participants in the IWOR, to all contributors to this issue and the numerous reviewers of the papers in this special volume for carefully reading and commenting on the papers. Their dedicated volunteer work has had a significant impact on the quality of the papers.. Finally, we would like to acknowledge the financial support of the Ministerio de Educación y Ciencia (grant MTM2007-30730-E), Universidad Rey Juan Carlos (Madrid, Spain), and Comunidad Autónoma de Madrid (Spain) that covered the expenses for the conference. 